\title{
Interpreter-Mediated Physician-Patient Communication: Opportunities for Multimodal Healthcare Interfaces
}

\author{
Nadir Weibel ${ }^{1}$, Colleen Emmenegger ${ }^{2}$, Jennifer Lyons ${ }^{2}$, Ram Dixit ${ }^{2}$, Linda L. Hill ${ }^{3}$, and James D. Hollan ${ }^{1,2}$ \\ ${ }^{1}$ Department of Computer Science and Engineering, ${ }^{2}$ Department of Cognitive Science \\ ${ }^{3}$ Department of Family and Preventive Medicine \\ University of California San Diego, La Jolla, CA 92093, USA \\ \{weibel, cemmenegger, j2lyons, rdixit, llhill, hollan\}@ucsd.edu
}

\begin{abstract}
Patient-centered health care and increased efficency are major goals of modern medicine. Research has shown that higher patient health literacy is linked to more successful health outcomes and the shift to electronic medical records (EMR) is hoped to increase efficency. Although EMR systems are designed to support physicians' clinical decision making, they can also hamper physician-patient communication, especially in the challenging conditions associated with interpreter-mediated interaction with low English proficiency patients. This paper examines EMR interaction and interpreter-mediated physician-patient communication. Based on a distributed cognition perspective that employs a novel methodology to simultaneously capture multiple data streams, we analyze multiparty and multimodal activity. The goal is to inform design of new multimodal healthcare interfaces.
\end{abstract}

\section{INTRODUCTION}

Medicine and healthcare are in the midst of a paradigm shift, rapidly moving away from an exclusive focus on treatment of disease towards a future of predictive and preventive personalized medicine enabled by identification of disease states before they produce serious symptoms. In this setting, the introduction of Electronic Medical Records (EMR) is changing the information environment of health care in the medical office. Programs to implement EMRs are being introduced in many countries. The US government, for example, is investing \$19.2 billion to encourage widespread EMR adoption by 2014. If EMRs are well designed and effectively used, they have the potential to improve patient health outcomes, patient safety, clinical efficiency, and patient-centered aspects of care [1]-[3]. While this has the potential to improve health care delivery and decrease costs, it is only one component in the very complex healthcare information ecology.

EMR systems are designed to aid information access, but they often introduce conflicts with current medical practices and can interfere with patient communication, potentially resulting in poorer medical outcomes. EMRs can shift the focus of the medical encounter from the patient to the computerized system [4], making the encounter less patient-centered. This impacts patient health literacy, leading to sub-optimal communication with patients and risks of limited patient understanding of procedure details, medications, treatments, etc. [5][7]. This is further complicated when language or cultural barriers are present, such as is common with non-native speaking patients who require use of interpreters [8]. This is a large and growing segment of the patient population. In the United States, over 320 languages are spoken by its residents ${ }^{1}$

\footnotetext{
${ }^{1}$ http://www.us-english.org/view/304
}

and nearly nine percent of the entire U.S. population speaks English less than "very well", while $20 \%$ of Californians are considered to have limited English proficiency (LEP). For many people, interpreters are essential to support physicianpatient communication and medical care.

Several studies suggest that usability problems in current EMRs may conflict with the intended system benefits and negatively impact medical practice [9], [10], introducing medical errors and even increasing patient mortality [11], [12]. Unless computerized health care systems are designed to meet the challenging requirements of productively coupling people and information systems, neither decreased costs nor improved health care delivery is likely to result. Therefore, design and implementation of EMRs should not be viewed as an end in itself. Rather, EMRs must give providers fast and accurate access to information without distracting them with burdensome documentation or inefficient user interfaces that impede clinical workflow.

Although previous studies have drawn attention to the need for studying EMR usability in complex clinical environments, significant knowledge gaps remain. Current work does not present an integrated comprehensive account of clinical work. As a result, it is difficult to understand the impact of the EMR on clinical activity. What is needed are careful analyses of how the EMR is used by physicians during patient encounters and how physicians and patients communicate. This is a necessary foundation for designing more effective EMR systems that not only take into account clinical workflow but also exploit emerging multimodal interface technologies.

In this paper, we present a study of interpreter-mediated physician-patient communication in which we analyzed activity involving multiple modalities of 12 patients interacting with a physician in a non-profit clinic serving low-income multicultural patients. A majority of the patients are LEP, and half require an interpreter to mediate communication with the physician.

\section{Communication in the Medical OfFice}

In order to study physician-patient communication we first need to understand the context and environment in which the activity is situated. In this paper we focus on communication and interaction in the medical exam room of a clinic. To position our work in the context of current research we summarize selected major themes of investigations of physician-patient communication and impacts of EMR use and interpretermediation on clincial workflow. 


\section{Communication influences Health Literacy}

The U.S. Institute of Medicine defines health literacy as "the degree to which individuals have the capacity to obtain, process, and understand basic health information and services needed to make appropriate health decisions." Health literacy is a key element of patient-centered health care, in which patients are encouraged to take a more active role in managing their own health and making informed decisions about medical interventions and treatments. Low health literacy impacts understanding and following clinician instructions, such as simple directions on how often to take a medication, the correct dosage, or whether to take it on a full stomach [13].

Influences on patient health literacy include individual knowledge, motivation, and the ability to use the information to promote their health [14]. Health literacy also depends on effective communication, on which we focus in this study. Research suggests that physician-patient communication is a critical influence on patient health literacy [15]. Schillinger reports that poor communication negatively impacts patients suffering from chronic diseases such as diabetes [13]. Other critical populations such as the elderly, and racial and ethnic patient populations are also affected [16]. Improving communication, especially for these populations, will improve patientcentered healthcare, leading to increased health literacy and better medical outcomes.

\section{Communication is Distributed and Multimodal}

Most research analyzing the use of technology in the medical field is based on classical models of cognition, focusing on the properties of single individuals. However, these models are limited in describing dynamic interaction and communication between individuals and with technology [17]. To accomplish this requires looking at individuals and technology as participants in systems and appreciating that the systems can have important emergent properties. The medical office is such a system, involving complex relationships between physicians, patients (who sometimes need interpreters), medical technology, and the surrounding physical environment.

The people, artifacts, and technology in the medical exam room constitute a dynamic distributed cognitive system [18][20]. Activity and communication in the exam room are distributed across physicians, patients, interpreters, artifacts, and technology. Additionally, multiple communication modalities are used. Traditionally, speech is considered the primary modality for communication, but nonverbal cues such as eye contact, gestures, and body orientation are also crucial aspects of the multiparty and multimodal nature of the distributed clinical medical activity.

\section{EMR Use Impacts Physician-Patient Communication}

Numerous studies, such as one reported by Frankel and colleagues [21], suggest that computer use in medical consultations often leads to a feeling of disengagement for patients, since for a large portion of time during the visit the physician's attention is directed at the computer rather than the patient. This may significantly reduce exchange of information, a critical aspect of the interaction and one that has been shown to correlate with medical information comprehension and judgements of positive patient-provider relationships [22].
Researchers have found that physician EMR screen gaze inhibits patients' "question asking and emotional responsiveness" [23]. In one observational study [24], five physicians were videotaped during patient visits in which EMRs were used. One consistent impact of EMR use was interference with the flow of physician-patient conversation.

\section{Interpreter Mediation Impacts Communication}

Although interpreters are essential in medical encounters for LEP patients, the impact of mediation by interpreters in medical settings has rarely been investigated nor considered in EMR design and evaluation.

Medical interpreters facilitate communication by translating between languages and cultures to aid information exchange between physicians and patients. This process includes not only language translation, but also acting as patient advocates [25] and helping negotiate cultural differences between healthcare providers and patients [26]. Inclusion of an interpreter changes the communication system. Communication is no longer an exchange solely between the physician and patient, but a mediated exchange between three parties. Communication is shaped by multiple conversations between the parties. This is a complex distributed system involving dynamic reconfiguations of attention and communication.

\section{EMR Use Impacts Clinical Workflow}

Studies suggest that EMRs introduce human-computer interaction problems, increase cognitive load, and alter the structure and dynamics of clinical activities [27]-[29]. A key aspect of clinical workflow is documentation of information. Such documentation is the basis not only for fulfilling clinical and legal requirements, but also creates opportunities to improve the quality of healthcare delivery and serves as a basis for education and secondary use of clinical data. However, complex tradeoffs are involved and benefits have to be balanced with considerations of work efficiency. Documentation consumes considerable time in clinical work, even more than direct patient care [30]. In one interview-based study [31], participants commented that computerized documentation can create new work processes that do not coincide with common clinical workflows and familiar practices. In fact, a systematic review [32] summarizing major studies in the computerized documentation area concluded that the goal of decreased documentation time is not likely to be realized through current EMR systems.

Heath and Luff [33] argue that although relevant categories of medical records are defined in the healthcare IT systems, the practices through which the document is written, read, and used within consultation have been largely ignored. The issue surrounding how medical records are used is closely related to how work is handled in complex clinical workflows and how information can be conveniently and unobtrusively documented. Chen [29] conducted a field study in an emergency room (ER) setting to examine EMR use in the complex flow of ER clinical processes. The study revealed that ER staff frequently rely on other artifacts such as paper notes to facilitate their work. Analysis of the use of these additional artifacts in four different clinical workflows demonstrates that EMR systems have clear limitations for supporting documentation of procedural information. 


\section{APPROACH}

As is clear from the above studies, communication in medical settings is a complex activity and designing technology to support the associated clinical workflow is extremely challenging. Our premise is that a necessary first step in meeting the myriad challenges involved is to approach these complex settings as distributed systems. To do this requires capturing data about communication and interaction between physicians, patients, and others involved in medical encounters.

Not enough is known about the real flow of communication in medical settings. Our approach is grounded in the theory of distributed cognition [18]-[20]. We use distributed cognition and methods of cognitive ethnography [34] to investigate medical settings as distributed systems. The goal is to better understand current practice as a foundation for designing technology to enhance performance in these settings.

Communication involves multiple parallel modalities. Although most previous work in medical human factors has examined single modalities involving single participants, we employ a suite of instrumentation and analysis techniques using ChronoViz [35], a tool that facilitates analysis of simultaneous bodily action, voice, gaze (head position) of multiple participants. Simultaneous analysis and visualization of the activity as enabled by our approach is key to really understanding the dynamics of this complex environment. Our approach allows us to document the distributed multiparty multimodal nature of clinical medical activity by capturing and analyzing data from entire clinical encounters. In addition, we automate significant aspects of the data collection, and visualization of the activity of the analyzed system.

\section{Methods and Participants}

We collected data in November 2012 at a local community health center which provides comprehensive medical care for low income and multi-ethnic patient populations. The center provides prenatal, pediatric, adult medicine, mental health, family planning, and outreach/community education services. It employs approximately 250 individuals, with 14-18 exam rooms at each of 3 sites. EMRs ${ }^{2}$ have been used for all patient encounters since May 2010. Chronic disease management is the main challenge among patients in the clinic. To support LEP patients, the clinic provides interpreters in 8 languages. They are always available to support physician-patient communication. Interpreters receive ongoing health education, and are often trained to function as medical assistants.

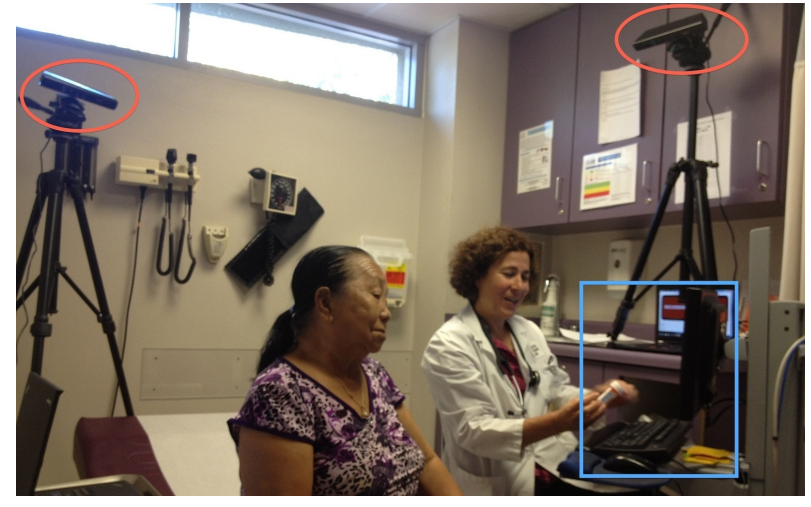

Fig. 1. Pilot installation at the local clinic. The Kinects are circled in red The computer used to enter and access EMR data is outlined in blue.
During our study the physician and patient sit side-byside. The physician sits in a rolling desk chair, with the EMR directly in front of her on a rolling, height-adjustable platform. The patient sits next to the physician on the front edge of a traditional exam table. When an interpreter is present, he/she commonly sits across the room, facing the physician and patient, or stands near the door. The interpreter typically sits in a simple chair backed against the exam room wall, next to the door, approximately six feet directly in front of the patient. Figure 1 shows the position of patient and doctor, while Fig. 2 (center) shows the position of the interpreter.

To capture multiparty multimodal activity we installed an experimental recording system using two Microsoft Kinects ${ }^{3}$ (Fig. 1). This allowed us to capture body positioning, directional audio, video footage, and depth-imaging of the scene. We recorded visits with 12 consented adult patients ( 7 female, 5 male, half requiring the service of an interpreter). We developed a dedicated Kinect-based tool to collect multimodal data in real-time and import it into ChronoViz. Each of the 12 data sets (one for each participant) contained two video streams, two directional audio streams, two depth data streams, and derived body joint positions (calculated by the Kinect algorithms). Session lengths varied from approximately 5 minutes to 13 minutes. The physician used a special pause button to stop recording during physical exams.

A group of 5 researchers analyzed the data from the 12 sessions, focusing on the general structure of the visit and occurrences of specific communication patterns. Given the richness and complexity of interaction between individuals and with the EMR, as well as the distinct physician-patient interactions while an interpreter was present in the room, two encounters were selected for detailed analysis. One session involved an English-fluent patient and the second was a session with an LEP patient requiring an interpreter. Body position, head position, right and left hand position, and speech instances (not transcriptions of them) of both patient and physician across the entire sessions were manually coded. Speech, body position, and head position were also annotated for the interpreter in the second session. Approximately 150 hours was required for this manual analysis and coding. Figure 2 shows the integrated data streams and the results of the coding of one session in ChronoViz. Inter-coder reliability was calculated for both multimodal activity identification and time span of the identified activity. Researchers agreed $100 \%$ on the classification when the activity was identified by all of them. Kappa Cohen's coefficient was calculated to identify missed activity (activities not identified by all coders) and reveled an accuracy of $86 \%$. Start time was identified with an average $393 \mathrm{~ms}$ variation. End time with an average variation of $1412 \mathrm{~ms}$. Activity length average variation was $1579 \mathrm{~ms}$.

\section{ANALYSIS}

After a first analysis pass of all sessions, an in-depth analysis of two selected sessions was performed and an annotated event record of type and duration of activity was constructed. This allowed identification of important characteristics and drove the evolving analysis scheme.

\footnotetext{
${ }^{2}$ http://www.eclinicalworks.com

${ }^{3}$ http://kinectforwindows.org
} 


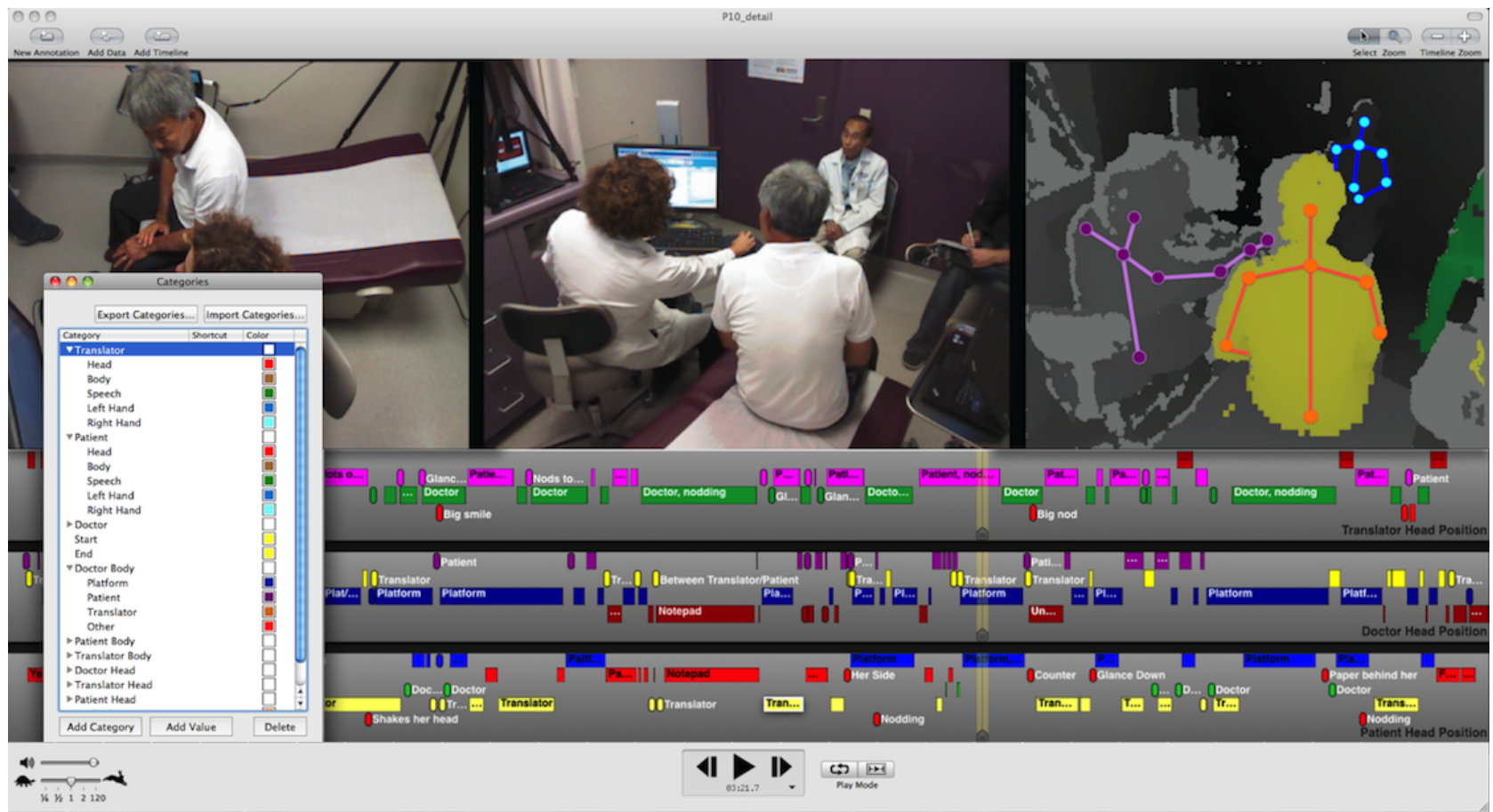

Fig. 2. ChronoViz view of Kinect data. The top half shows video feeds (two video and one depth-image) from two Kinects. The center video and right depth-image show the interpreter facing the physician and patient. The physician sits left and the patient sits on the exam table. The bottom half shows three timelines with annotations of a 5 minute medical session. These timelines can indicate information such as who is talking (patient, interpreter, doctor), their body positions and what they are interacting with. In this figure, we see annotations indicating head direction for each of the three participants. The video frames are associated with the time indicated by the vertical yellow bar. The coding categories are seen in the overlaid window at left.

The system included the following elements:

(a) Individuals: patient, physician, medical assistant, interpreter, family member

(b) Artifacts: tables (exam table, doctor's mobile station), chairs (doctor's, relative's and/or interpreter's chair), EMR system, paper documents (brought by clinical staff, the patient and/or already in the exam room), other artifacts (prescription bottles brought in by a patient).

(c) Multimodal Communication: speech (discourse by the patient, physician, interpreter, patient family, and medical assistant), hands (left and right hands of physician, patient, and interpreter), movements (includes writing, typing, touching, holding), body (position of all people in the room, including leaning, standing, sitting, etc.), head (position of physician, patient and interpreter), gestures (include touching body part, indicating to EMR, etc.).

(d) Socio/Cultural Resources: medical knowledge, language knowledge (English, foreign languages, etc.), cultural knowledge.

\section{Speech, Gesture and Body Communication Patterns}

Detailed analysis revealed differing multiparty communication patterns. One simple example is verbal communication between the physician, patient and interpreter. Figure 3 illustrates a timeline showing the interpreter functioning as a middle man in the communication. The interpreter speaks directly after both the patient and the physician. As we discuss below, this pattern is only interrupted when the interpreter is not able to directly translate the doctor's or patient's speech due to usage of other artifacts (e.g., paper, EMR).

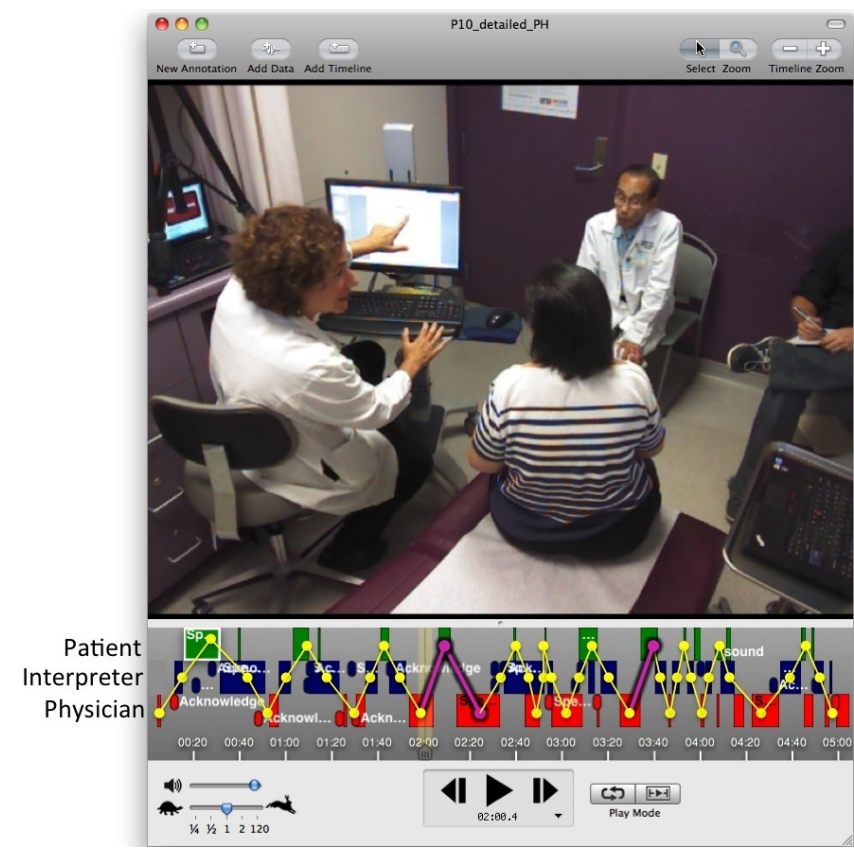

Fig. 3. Speech Analysis of an interpreter-mediated communication session. The timeline displays three levels, each representing the speech of one member of the team: the top line is the patient's speech (green), the middle line is the interpreter's speech (blue), and the lowest line displays the physician's speech (red). A line is superimposed to connect utterances of the three individuals Yellow lines represent common physician-interpreter-patient interaction patterns, purple lines identify the rare physician-patient interaction patterns.

Verbal communication patterns differ between interpreter and non-interpreter sessions. The physician and patient spoke less $(35 \%$ and $25 \%$ respectively) than the interpreter (more than $40 \%$ ). When no interpreter was present, physician and 

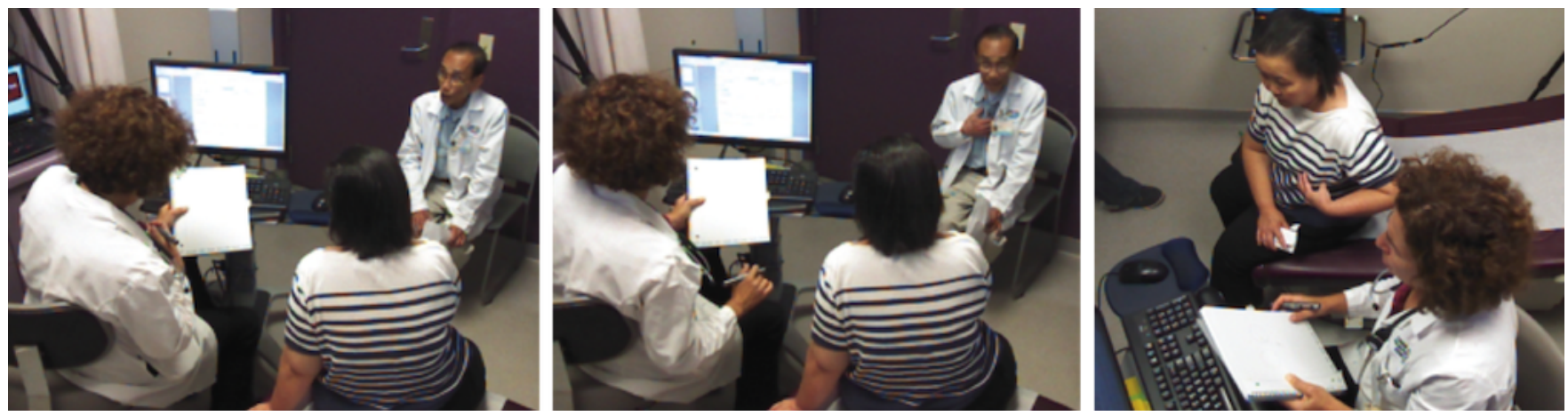

Fig. 4. Interpreter-mediated gestures. This triptych represents a temporal sequence of a gesture performed first by the physician to indicate the location of a lump in a particular region, repeated by the interpreter, and finally repeated by the patient.

patient each spoke approximately $50 \%$ of the time. In this study, sessions with an interpreter present had an approximate mean length of 7.79 minutes (sd 1.80) and sessions without an interpreter present had an approximate mean length of 8.56 minutes (sd 0.74). For sessions of approximately the same length, this may result in less time for a patient with an interpreter to describe problems, ask questions, etc. as well as for the doctor to respond to queries, troubleshoot medical issues, discuss prevention, etc.

Gesture communication patterns also differed between the interpreter and non-interpreter sessions analyzed in detail. In both sessions, a variety of gesture types were observed, including deictic (e.g. pointing at EMR or paper), iconic (e.g. hand in shape of cyst) and beat gestures (e.g. hand palm up). When no interpreter was present, the physician's gestures were distributed fairly evenly across the three gesture types (approximately $39 \%$ deictic, $36 \%$ iconic, $24 \%$ beat, as percentages of overall gesture types), and the patient communicated with more beat gestures (approximately 36\% deictic, 10\% iconic, 55\% beat). The presence of the interpreter radically changed the gesture pattern: the physician used iconic gestures much more often (approximately 31\% deictic, $44 \%$ iconic, $25 \%$ beat), while all of the interpreter's and patient's gestures were iconic (gesture were used exclusively to communicate the shape, size and location of an injury). An interesting transitive iconic gesture pattern propagating through the trio of participants also emerged in the interpreter's session. Figure 4 illustrates one such gesture pattern example.

\section{Communication Pattern Breakdown}

Visualization of the annotated record of interaction highlighted instances of communication breakdown. One indication of a breakdown was the interruption of the interpreter's middleman pattern of the speech mentioned above. For example, we saw this pattern disrupted when the physician was attempting to explain a test result to a patient that had been misinterpreted. The physician first turns the EMR display towards the patient and points to some information on the screen. She then pulls out a notebook, holds it so the patient can see it and begins to draw. While drawing, she speaks and gestures toward the paper. The interpreter is left out of this interaction and cannot see the referencing artifacts. In the annotated record this is evidenced by disruption of the alternating pattern of speech. Four communication breakdowns occurred during three physician-patient encounters where an interpreter was being heavily utilized.

\section{EMR as Communication Tool}

EMR systems are designed primarily to assist physicians in documentation. In addition, they help reduce drug interactions and provide information about other potential problems by providing alerts. When the boundary of the unit of analysis is expanded to include all participants in a shared exam room, we can identify additional usage patterns. Physicians frequently refer to the information on the EMR screen to assist in communicating with patients. Figure 5 illustrates two examples. On the left, the physician and patient were discussing poor control of his blood sugar level. During the discussion, he was asked if he had seen the diabetes education specialist recently. The patient replied that he had, "many times." From the EMR data, the physician sees the patient had not actually seen a specialist for two years, having missed two previous appointments. The physician immediately points this out to the patient by referencing the EMR screen. The patient then acknowledges his error and agrees to see the diabetes specialist immediately after the session.

The image in the right panel of Fig. 5 illustrates another example. The physician is using the EMR system to involve the patient in collaboratively verifying current medical prescription information. The physician and patient are jointly referring to the EMR screen while identifying the medications the patient was currently taking, and whether they need to be refilled. The patient, sitting on the exam table, uses a paper document in her hand to indicate which medications she wanted.

\section{Paper Artifact Use}

Paper is a commonly used artifact, often brought in by clinic staff, patients, or already present in the exam room. The interpreter and other staff members frequently use sticky notes, containing patient names and vitals. Figure 6 , left panel, shows the interpreter holding a sticky note. These are frequently placed on the corner of the mobile computer platform to help confirm patient identity and that the correct chart was open on the EMR. Interestingly, although the EMR displays the name and a photograph of the patient, the photograph quality is not sufficient to make a positive patient identification.

As mentioned earlier, sometimes physicians use the EMR to support communication with patients (e.g., to help explain a test result). However, often to fully explain a concept, the physician pulled out a notebook (see Fig. 4) and drew a picture to help communicate information she was attempting to share with the patient. In addition, patients often brought information 

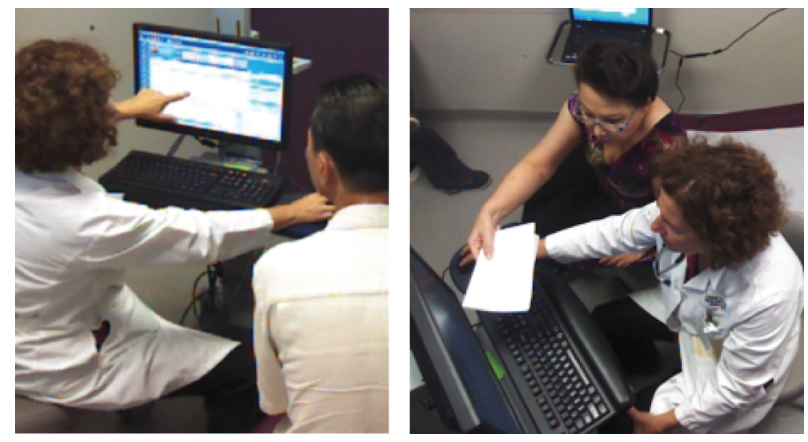

Fig. 5. Interaction with the EMR. Left: the physician directs the patient towards the EMR with a pointing gesture. Right: the patient directs the paper document she has brought with her to the consultation towards the EMR.

on sheets of paper. This included results from laboratory tests, off-site procedures, medication refills, or recent blood sugar levels (see right image of Fig. 6). These documents are often central to discussions. For example, one patient brought lab results from a recent off-site CT scan and had questions about the results. The physician wanted to add the results to the EMR, so she put the sheet aside for the medical assistant to scan and import into the EMR at a later time.

\section{DISCUSSION}

Although modern EMRs are designed to support clinical documentation and help medical providers to make data-driven decisions, they also impact the flow of communication between physician and patient, and can interfere with it. There are additional challenges with non-native speakers who need an interpreter to support communication and understanding. The data reported here, and the view of the medical clinic as a distributed system, provide a basis for some initial suggestions for better supporting communication.

\section{Someone is often in the dark}

From observing patterns of communication it is clear there are many challenges and opportunities to improve communication. One observation is that often one of the parties involved is "left in the dark." This is especially the case with interpreter-mediated communication. The patient can't understand what the physician is saying and must await the interpreter's translation and the physician can't understand what the interpreter is saying to the patient nor be sure of translation accuracy. In addition, there are challenges of where to direct attention when various parties are talking. We know that much is conveyed by facial expressions and gesture, but the patient may be looking at the interpreter when the physician is talking or the physician at the interpreter when the patient is talking and as a result miss important cues. The communication process is particularly challenged when the interpreter is left out of the loop because of inability to attend to the EMR display or some other artifact, such as paper.

There were multiple instances in which the physician attempted to communicate directly with the patient through use of graphical aids. She drew pictures on a piece of paper or pointed to the EMR, but the patient was still unable to understand without the assistance of the interpreter. The interpreter was unable to see the visual aid, making it difficult for him to convey the full meaning of what the physician was attempting to communicate.
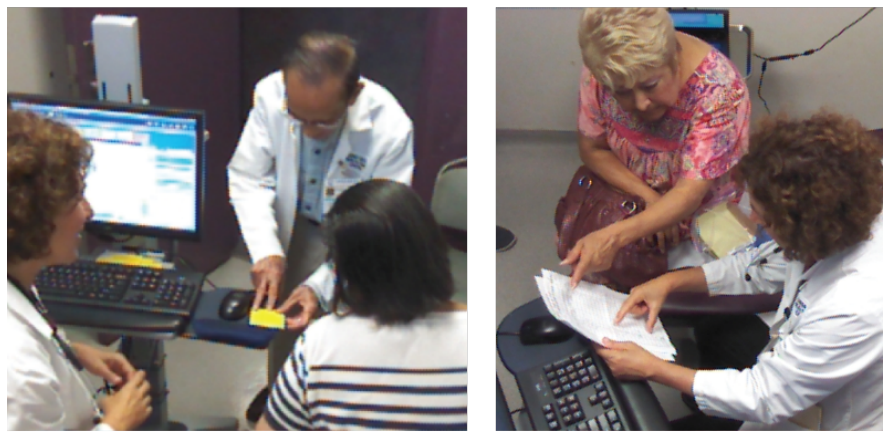

Fig. 6. Interaction with paper documents. Left: the interpreter points to a special sticky note to introduce a new patient. Right: the patient discuss with the physician her blood sugar levels noted on a paper document she brought.

\section{The EMR fails as a shared communication tool}

The information available on the EMR is potentially valuable in helping the patient understand diagnoses, treatments, medication, etc. The physician pointing to the information displayed on the EMR was a common occurrence and our analysis of gestures and body position confirm that patients seemed interested in looking at this data. A key problem however, is that neither the patient nor the interpreter can effectively see the display. Studies [36], [37], report that the dimensions, resolution, and arrangement of the display restrict access primarily to the physician sitting at the computer.

Our analysis also shows that the effectiveness of the EMR as a shared communication tool changed with the introduction of an interpreter. While sessions with native speaker patients show the physician and patient pointing at the EMR in equal ways, when an interpreter was present no pointing gestures to the EMR by the patient or the interpreter was identified. This suggests the EMR was even less effective for LEP patients.

\section{Implications for design}

We see considerable potential for the EMR display to function as an important communication resource. Currently only limited attention has been given to design of the EMR interface, especially in terms of aiding physician-patient communication. In the HCI research community, numerous effective technologies to support tangible interaction with digital information have been introduced over the last 15 years [38][40], but their potential to assist medical applications has not been explored nor evaluated. For example, multitouch technologies enable interaction with information on tabletop displays or projected onto desktops [41]. Technologies for tracking body, hands, and fingers [42], [43] or pen and paper activities [44], [45] support interaction in hybrid physicaldigital environments common to most medical and other office settings. Moreover, well-established findings from the computer-supported cooperative work community (CSCW) and from our own lab [46] demonstrate that positioning participants around table-like surfaces facilitates face-to-face group interaction [47].

There is an exciting opportunity to exploit modern multimodal interaction technology and flat surfaces to provide hybrid digital-physical information spaces. These spaces would not only support natural interactions, but have the potential to improve communication between patients and medical professionals [46]. They would shift the common 
interaction paradigm, currently characterized by separate patient-physician, interpreter-patient, and physician-EMR interactions, to a richer interaction across patient, interpreter, physician, and EMR. Such interaction would improve communication and increase patients' involvement in their healthcare.

We envision exploiting our current research on mobile projected interactive surfaces based on Microsoft Kinect and short-throw projectors [48] to create an augmented tabletop experience employing a projected surface and sensors to track touch-based interactions and mid-air gestures. Physicians could select information from the EMR that they want to share with the patient (and the interpreter) and visualize it in the shared space. To minimize installation issues and accommodate limited medical office space, existing surfaces such as desks and walls could be exploited. Additionally, equipment with a flip-out surface could be installed on the physician's mobile station for use where and when needed. These facilities would allow physicians, patients, and interpreters to interact with shared projected information. In addition to standard touch and gesture-based interaction, the interface could also capture any paper-based information (e.g., the blood sugar chart filled in at home), specific samples (e.g., a particular skin problem on the hand of a patient), or project a short instructional video. The physician could also activate a microphone and record audio samples (e.g. of a patient with a speech impairment). This information should be incorporated into the EMR system.

\section{FUTURE WORK}

Understanding the patterns of communication and interaction within the distributed system of the medical office is essential for designing effective new multimodal interfaces to support physician-patient communication. This understanding is especially valuable when designing for LEP patients. While the analysis reported here is only a beginning basis for our design activities, we think a major contribution is the identification of the crucial importance of taking a system perspective and looking closely at the medical encounter as it evolves in the interaction among individuals, artifacts, and technologies.

There are many challenges for the future. Not the least of which is the complexity of analyzing rich data from natural settings. Our team spent more than 150 hours over 7 weeks to begin to analyze data from 12 short medical encounters. We need automatic and semi-automatic annotation facilities to help with the multiple data streams recorded with the Kinect devices. Currently we are exploring extensions of the ChronoViz tool to identify specific behaviors and automatically generate annotations on ChronoViz timelines. One example is integrating external tools for speech signal and conversational analysis that exploit the directional audio recorded by the Kinect to identify the speech of single individuals in the room and generate visualizations of who is talking during interactions. In addition, this will allow us to compute the pattern of interaction and quantitative measures of the amount of speaking time for each individual.

We are also enhancing the skeleton tracking facility of the Kinect to automatically generate annotations for gestures of particular interest, such as hand movements or pointing gestures (e.g., to the EMR). The combination of speech tracking and body tracking can then automatically generate statistics of the relative position of participants' bodies as well as infer gaze direction and identify specific types of interactions (e.g., faceto-face). Finally, we are planning to exploit the new facilities made available by KinectFusion [49] to automatically create $3 \mathrm{D}$ visualizations of interactions in the medical office space.

These automatic and semi-automatic data annotation facilities will advance analysis and accelerate design, deployment, and evaluation of new multimodal healthcare interfaces. In this setting we plan to evolve our current software framework for interaction on and above the desk surface [48]. We are working with a team of physicians and information visualization, informatics, and HCI researchers to investigate how novel interface technology can be integrated into clinics such as the one presented in this study. We plan to use the next generation of our data integration and analysis tools to continue to collect and analyze data in a variety of medical office situations to inform the design and deployment of future interfaces.

\section{CONCLUSION}

Effective interfaces are essential enablers for fully exploiting information technology to improve future health care. We see new opportunities to improve design of EMRs, provide more effective communication in the medical office, and increase health literacy and positive medical outcomes. The study of interpreter-mediated patient-physician communication presented in this paper illustrates current issues confronting EMR systems and describes our approach to understanding and impacting health communication and literacy. We highlight a new methodology for capturing and analyzing communication in the medical office. This methodology will aid researchers in understanding multimodal and multiparty interaction, how technology can support natural multimodal interfaces for medical information in the post-desktop future, and improve interface design of future health care systems.

Realizing this future presents major research challenges for many disciplines, especially for human-computer interaction. The premise of this paper is that radically improving humancomputer interaction is fundamental to the success of future health information technology. Fully capitalizing on the potential of this technology to improve health care necessitates advances in theory and analytical methods required to address the complex challenges of designing future distributed multimodal information environments and creating a foundation for human-centered and data-driven interface development. As we have shown here, nowhere are these challenges clearer or more pressing than in interface design for EMRs and for visualization and interaction with complex health data.

\section{REFERENCES}

[1] Committee on Quality of Health Care in America, Institute of Medicine (National Academy Press), "Crossing the quality chasm: a new health system for the twenty-first century," 2001.

[2] R. Hillestad, J. Bigelow, and A. Bower, "Can electronic medical record systems transform health care? potential health benefits, savings, and costs," Health Aff (Millwood), vol. 24(5), pp. 1103-17, 2005.

[3] S. Wang, B. Middleton, and L. Prosser, "A cost-benefit analysis of electronic medical records in primary care," Am J Med., vol. 114(5), pp. 397-403, 2003.

[4] N. Shaw, "Medical education \& health informatics: time to join the 21 st century?" Studies in health technology and informatics, vol. 160 (Pt. 1), pp. 567-571, 2010. 
[5] D. W. Baker, J. A. Gazmararian, M. V. Williams, T. Scott, R. M. Parker, D. Green, J. Ren, and J. Peel, "Functional health literacy and the risk of hospital admission among medicare managed care enrollees," American Journal of Public Health, vol. 92, no. 8, pp. 1278-1283, 2002.

[6] D. Baker and M. Wolf, "Health literacy and mortality among elderly persons," Arch Intern Med, vol. 167(14), pp. 1503-1509, 2007.

[7] A. R. Wittich, J. Mangan, R. Grad, W. Wang, and L. B. Gerald, "Pediatric asthma: Caregiver health literacy and the clinician's perception," Journal of Asthma, vol. 44, no. 1, pp. 51-55, 2007.

[8] G. Flores, "The impact of medical interpreter services on the quality of health care: A systematic review," Medical Care Research and Review, vol. 62, no. 3, pp. 255-299, 2005.

[9] S. Rosenbloom, F. J. Harrell, C. Lehmann, J. Schneider, S. Spooner and K. Johnson, "Perceived increase in mortality after process and policy changes implemented with computerized physician order entry," Pediatrics, vol. 117(4), pp. 1452-6, 2006.

[10] A. Virapongse, D. Bates, and P. Shi, "Electronic health records and malpractice claims in office practice," Arch. Intern Med., vol. 168(21), pp. 2362-7, 2008.

[11] Y. Han, J. Carcillo, and S. Venkataraman, "Unexpected increased mortality after implementation of a commercially sold computerized physician order entry system," Pediatrics, vol. 116(6), pp. 1506-12, 2005.

[12] R. Koppel, J. Metlay, and A. Cohen, "Role of computerized physician order entry systems in facilitating medication errors," JAMA, vol 293(10), pp. 1197-203, 2005.

[13] D. Schillinger and K. Grumbach, "Association of health literacy with diabetes outcomes," JAMA, vol. 288, no. 4, pp. 475-482, 2002.

[14] A. Peerson and M. Saunders, "Health literacy revisited: what do we mean and why does it matter?" Health Promotion International, vol. 24, no. 3, pp. 285-296, 2009.

[15] M. Williams, T. Davis, R. Parker, B. Weiss et al., "The role of health literacy in patient-physician communication," Family Medicine Kansas City, vol. 34, no. 5, pp. 383-389, 2002.

[16] M. Mueller, S. Roussos, L. Hill, N. Salas, V. Villarreal, N. Baird, and M. Hovell, "Medical interpreting by bilingual staff whose primary role is not interpreting: Contingencies influencing communication for dual-role interpreters," Access to Care and Factors that Impact Access, Patients as Partners in Care and Changing Roles of Health Providers, vol. 29, pp. 77-91, 2011.

[17] B. Hazlehurst, P. Gorman, and C. McMullen, "Distributed cognition: an alternative model of cognition for medical informatics." International journal of medical informatics, vol. 77, no. 4, p. 226, 2008.

[18] E. Hutchins, Cognition in the Wild. Cambridge: MIT Press, 1995.

[19] J. Hollan, E. Hutchins, and D. Kirsh, "Distributed cognition: toward a new foundation for human-computer interaction research," ACM Trans. Comput.-Hum. Interact., vol. 7, no. 2, p. 174196, 2000.

[20] J. D. Hollan and E. L. Hutchins, "Opportunities and challenges for augmented environments: A distributed cognition perspective," in $\mathrm{De}$ signing User Friendly Augmented Work Environments: From Meeting Rooms to Digital Collaborative Spaces, S. Lahlou, Ed. Springer, 2009.

[21] R. Frankel, A. Altschuler, and S. George, "Effects of exam-room computing on clinician-patient communication: a longitudinal qualitative study," J. Gen. Intern. Med., vol. 20(8), pp. 677-682, 2005.

[22] G. Makoul, R. Curry, and P. Tang, "The use of electronic medical records: communication patterns in outpatient encounters," J. Am. Med. Inform. Assoc., vol. 8(6), pp. 610-615, 2001.

[23] R. Margalit, D. Roter, M. Dunevant, S. Larson, and R. S., "Electronic medical record use and physician-patient communication: an observational study of israeli primary care encounters," Patient Educ. Couns., vol. 61(1), pp. 134-141, 2006

[24] A. Als, "The desk-top computer as a magic box: patterns of behaviour connected with the desk-top computer; gps' and patients' perceptions,' Fam. Pract., vol. 14(1), pp. 17-23, 1997.

[25] E. Hsieh, "I am not a robot! interpreters' views of their roles in health care settings," Qual Health Res, vol. 18(10), pp. 1367-1383, 2008.

[26] D. Dysart-Gale, "Clinicians and medical interpreters: Negotiating culturally appropriate care for patients with limited english ability," Family \& community health, vol. 30, no. 3, pp. 237-246, 2007.
[27] C. Weir, J. Nebeker, B. Hicken, R. Campo, F. Drews, and B. Lebar, "A cognitive task analysis of information management strategies in a computerized provider order entry environment," J. Am. Med. Inform. Assoc., vol. 14(1), pp. 65-75, 2007.

[28] Y. Chen, V. Ngo, S. Harrison, and V. Duong, "Unpacking exam-room computing: negotiating computer-use in patient-physician interactions," in Proc. CHI' '11, 2011, pp. 3343-3352.

[29] Y. Chen, V. Ngo, S. Harrison, and V. Duong, "Documenting transitional information in EMR," in Proc. CHI '10, 2010, pp. 1787-1796.

[30] A. Hendrich, M. Chow, B. Skierczynski, and Z. Lu, "A 36-hospital time and motion study: how do medical-surgical nurses spend their time?" Perm. J., vol. 12(3), pp. 25-34, 2008.

[31] P. Embi, T. Yackel, J. Logan, J. Bowen, T. Cooney, and P. Gorman, "Impacts of computerized physician documentation in a teaching hospital: perceptions of faculty and resident physicians," J. Am. Med. Inform. Assoc., vol. 11(4), pp. 300-9, 2004.

[32] L. Poissant, J. Pereira, R. Tamblyn, and Y. Kawasumi, "The impact of electronic health records on time efficiency of physicians and nurses: a systematic review," J. Am. Med. Inform. Assoc., vol. 12(5), pp. 505-16, 2005.

[33] C. Heath and P. Luff, "Documents and professional practice: bad organizational reasons for good clinical records," in Proc. CSCW '96, 1996, pp. 354-363.

[34] E. Hutchins, "Cognitive ethnography," Plenary address at the 25th meeting of the Cognitive Science Society, Boston, 2003.

[35] A. Fouse, N. Weibel, E. Hutchins, and J. D. Hollan, "ChronoViz: a system for supporting navigation of time-coded data," in Proc. CHI '11, 2011, http://chronoviz.com.

[36] T. Rodden, Y. Rogers, J. Halloran, and I. Taylor, "Designing novel interactional workspaces to support face to face consultations," in Proc. CHI' 03,2003 , pp. 57-64.

[37] M. Scaife, J. Halloran, and Y. Rogers, "Let's work together: supporting two-party collaborations with new forms of shared interactive representations," Cooperative systems design: A challenge of the mobility age, vol. 74, p. 123, 2002.

[38] H. Ishii and B. Ullmer, "Tangible bits: Towards seamless interfaces between people, bits and atoms," in Proc. CHI '97, vol. 1, 1997, pp. 234-241.

[39] S. R. Klemmer, J. Li, J. Lin, and J. A. Landay, "Papier-mâché: toolkit support for tangible input," in Proc. CHI '04, 2004, pp. 399-406.

[40] S. Hodges, S. Izadi, A. Butler, A. Rrustemi, and B. Buxton, "ThinSight versatile multi-touch sensing for thin form-factor displays," in Proc. UIST '07, 2007, pp. 259-268.

[41] M. Weiss, J. Wagner, Y. Jansen, R. Jennings, R. Khoshabeh, J. D. Hollan, and J. Borchers, "SLAP widgets: Bridging the gap between virtual and physical controls on tabletops," in Proc. CHI '09, 2009, pp. 481-490.

[42] C. Harrison, H. Benko, and A. D. Wilson, "OmniTouch: wearable multitouch interaction everywhere," in Proc. UIST '11, 2011, pp. 441450.

[43] A. D. Wilson, "Using a depth camera as a touch sensor," in Proc. ITS '10, 2010, pp. 69-72.

[44] M. C. Norrie, B. Signer, and N. Weibel, "General framework for the rapid development of interactive paper applications," in Proc. CoPADD '06, 2006, pp. 9-12.

[45] F. Guimbretière, "Paper augmented digital documents," in Proc. UIST '03, 2003.

[46] A. M. Piper and J. D. Hollan, "Supporting medical conversations between deaf and hearing individuals with tabletop displays," in Proc. CSCW'09, 2008, pp. 147-156.

[47] M. Morris, "Supporting effective interaction with tabletop groupware," in Proc. TableTop '06, 2006, pp. 55-56.

[48] Y. Liu, N. Weibel, and J. D. Hollan, "Interactive Space: A Framework for Prototyping Multitouch Interaction On and Above the Desktop," in Proc. CHI '13, 2013, in press.

[49] S. Izadi, D. Kim, O. Hilliges, D. Molyneaux, R. Newcombe, P. Kohli, J. Shotton, S. Hodges, D. Freeman, A. Davison, and A. Fitzgibbon, "Kinectfusion: real-time $3 \mathrm{~d}$ reconstruction and interaction using a moving depth camera," in Proc. UIST '11, 2011, pp. 559-568. 